\title{
Radiation Therapy for Patients with Thymoma: When, Where and How?
}

\author{
Aruna Turaka* \\ Assistant Professor, Department of Radiation Oncology, Allegheny Health Network, 320 East North Avenue, Pittsburgh PA 15212, USA
}

*Corresponding author: Aruna Turaka, Assistant Professor, Department of Radiation Oncology, Allegheny Health Network, 320 East North Avenue, Pittsburgh PA 15212, USA, Tel: + 412-359-3358; Fax: + 412-359-3981; E-mail: aturaka@wpahas.org

Received date: Jul 07, 2014, Accepted date: Jul 17, 2014, Publication date: Jul 25, 2014

Copyright: (c) 2014 Turaka A, et al. This is an open-access article distributed under the terms of the Creative Commons Attribution License, which permits unrestricted use, distribution, and reproduction in any medium, provided the original author and source are credited.

\section{Editorial}

\section{Introduction}

Thymomas are very sensitive to radiation therapy and chemotherapy. Current treatment involves multidisciplinary management including surgery, chemotherapy and radiation therapy. The most important prognostic factors after resection are Masaoka stage, World Health Organization (WHO) histology, complete resection status and size of the lesion. Maintaining a long-term disease-free survival for patients with locally advanced disease is important as most of the lesions are locally invasive with less malignant potential or lower rates of metastatic spread.

\section{When?}

Radiation therapy (RT) helps to improve the local control rates and survival. Radiation therapy is used as [1]. Adjuvant therapy for stage I and II patients; [2]. As neoadjuvant treatment with or without chemotherapy in medically operable locally advanced cases; [3]. Definitive radiation for unresectable patients with stages III/Iva disease, and 4 . As palliative therapy for symptomatic, metastatic sites.

\section{Where?}

Stage I patients after R0 resection are usually observed with interval follow ups and serial imaging studies. Role of radiation therapy for stage II is controversial and is recommended for patients with pleural invasion (T2b disease), close $(<1 \mathrm{~mm})$ surgical margin, higher grade (WHO B3), and tumours with invasion of the surrounding tissues after resection (R1). In patients with stage III/IVa disease with sub-total resection $(R 1 / R 2)$, adjuvant radiation therapy helps to reduce the local recurrence (LR).

A prospective randomized study was done in China for stage I patients with thymoma in 29 patients and randomized to observation after surgery and radiation therapy showed no difference in outcomes in recurrence patterns, metastases [1]. Majority of patients were less than 65 years of age and AP/PA with oblique wedge pair techniques were used for RT planning. For lymphocyte predominant histology, RT dose of $50 \mathrm{~Gy}$ in 25 fractions was given and epithelial/mixed variants were treated to $60 \mathrm{~Gy}$ in 30 fractions. The 10 -year overall survival (OS) in the surgery alone group was $92 \%$ and $88 \%$ for patients treated with adjuvant RT.

Role of RT for stage II patients is considered controversial but several published single institution series have shown decrease in local control rates in patients treated with adjuvant radiation therapy compared with observation for stage II patients. A multi-institutional retrospective review of 103 cases was done by Ogawa et al about the role of postoperative radiation therapy in completely resected thymoma cases (Masaoka stage I in 17\%, 59\% stage II and 24\% with stage III disease). None of the patients were treated with chemotherapy [2]. The median RT dose was 40 Gy with $51 \%$ treated with involved field RT and $49 \%$ treated to the entire mediastinum. Median follow up was 9.3 years. The 10 -year OS for the entire cohort was $81 \%$ with $100 \%$ for stage I, $90 \%$ for stage II and $48 \%$ for stage III. None of the patients had in-field recurrences and $70 \%$ recurrences were within the pleura. For patients with pleural invasion noted at diagnosis, $38 \%$ had pleural failures. No correlation was noted with RT doses either above or $\leq 40$ Gy and authors felt that 40 Gy helped to reduce the recurrences in patient with pathological pleural invasion. Comparison of outcomes was done for stage I \& II patients treated with adjuvant RT in 167 patients from University of Pennsylvania by Singhal et a [3]. Of these, 70 patients were stage IIb treated with surgery \pm RT. An RT dose of 45-55 Gy in 25-33 fractions was given for 23 patients. At a median follow up of 70.3 months, local recurrence was noted in $1.4 \%$ with one patient in each surgery and RT groups noticing LR. There was no difference in OS with a 5 year-OS of $91 \%$. An updated subset analysis in high-risk stage II patients after complete resection was done by Berman et al in 175 patients treated between years 1990- 2008 from University of Pennsylvania [4]. Complete resection was done in 62 patients and 37 had high-risk features treated to a dose of $50.4 \mathrm{~Gy}$. Median follow up was 52 months and the overall LR rate was 3.2\% (8.3\% after surgery and $0 \%$ after RT, $\mathrm{P}=0.15$ ). Impact of adjuvant RT on OS and cause-specific survival (CSS) was done in 1464 patients from SEER database [5] by Suntharalingam et al. Median follow up was 41 months. Median OS for patients treated with surgery alone was 80 months and 97 months for those treated with adjuvant RT. The 10year OS for the total cohort was $42 \%$ treated with RT, $41 \%(\mathrm{p}=0.06)$ without RT and the 10 year CSS rates were $72 \% \& 76 \%(\mathrm{p}=0.85)$. For patients with incomplete resection, the outcomes were: 10 -year OS was $63 \%, 46 \%(\mathrm{p}=0.4)$ and CSS was $81 \%, 80 \%(\mathrm{p}=0.9)$. Addition of adjuvant RT was correlated with improvement in 10 year OS for stage II-III patients $41 \%$ compared with $35 \%$ without RT $(\mathrm{p}=0.002)$ but there was no difference in CSS. A pooled data6 from three institutions was reviewed by Curran et al. [6] about the role of mediastinal radiation therapy after complete/incomplete resection in 103 patients treated between 1960-1985. Five- year OS was $67 \%$ for stage I, $86 \%$ for stage II, $69 \%$ for stage III, with a relapse free survival of $100 \%, 58 \%$ and $53 \%$ for each stage. For stage II-III patients after R0 resection, the LR was noted in 53\% without RT and $0 \%$ in patients treated with RT. LR for the entire cohort was $28 \%$ without RT and $5 \%$ with RT.

\section{How?}

Recent trends in radiation therapy for thymoma: Advancements of radiation therapy techniques will have a large impact on reducing serious morbidity. Introduction of 3-dimensional conformal techniques (3D CRT) compared with 2D techniques will help to select the optimum beam angles to shape the treatment field, while 
Citation: Turaka A (2014) Radiation Therapy for Patients with Thymoma: When, Where and How?. J Nucl Med Radiat Ther 5: e111. doi:

Page 2 of 2

minimizing dose to the critical structures. Long-term toxicities were noted in $5-15 \%$ of cases with the use of $2 \mathrm{D}$ RT techniques which is usually unacceptable as these individuals have longer survival rates [7]. Intensity modulated radiation therapy (IMRT) also helps to optimize the dose to the tumour and the normal tissues, delivers non-uniform dose to the tumour within the RT field, improving dosimetric conformity and thus the therapeutic ratio. Application of proton therapy provides a sharp increase in dose at a given depth in the tissue. Rigorous treatment planning techniques helps to analyze the doses to the critical structures by following the strict dose-volume histogram $(\mathrm{DVH})$ criteria. $4 \mathrm{D}$ treatment planning helps to measure the tumour motion and define the internal target volume (ITV). Various image guidance tools in the form of cone-beam CT (CBCT) also helps to reduce the treatment volumes. In the pre-CT era, the target volume was defined as gross tumour volume (GTV), bilateral mediastinal lymph nodes and bilateral hilar nodes and/or mediastinum without including the hilum. Currently, clinical target volumes (CTV) are confined to the pre-surgical extent of disease, to include the entire surgical bed without elective nodal radiation except for thymic carcinoma cases. A dose of 45-50.4 Gy given in 180-200 cGy per fraction over 5-6 weeks duration in a postoperative situation with clear/negative margins, $54 \mathrm{~Gy}$ for positive margins, 60-70 Gy for gross residual disease.

Adaptive radiation therapy techniques are emerging which helps to make changes in the treatment based on the tumor response, inflammatory changes related to treatment, breathing patterns and alterations in patient weight during the treatment. Modern series are incorporating stereotactic radiation therapy (SBRT) as boost in patients with bulky, unresectable tumours.

\section{Conclusions}

Surgery is the standard of care for the early stage disease. Role of radiation therapy is controversial in stage II patients but in select patients with high-risk features it has been shown to decrease the local recurrence rates. Radiation therapy can be safely given in unresectable patients with the use of modern RT techniques including IMRT with image guidance (IGRT). Use of adaptive RT techniques will also help to reduce the dose to the normal critical structures and thus reduce the incidence of late side effects.

\section{References}

1. Zhang H, Lu N, Wang M, Gu X, Zhang D (1999) Postoperative radiotherapy for stage I thymoma: a prospective randomized trial in 29 cases. Chin Med J (Engl) 112: 136-138.

2. Ogawa K, Uno T, Toita T, Onishi H, Yoshida H, et al. (2002) Postoperative radiotherapy for patients with completely resected thymoma: a multiinstitutional, retrospective review of 103 patients. Cancer 94: 1405-1413.

3. Singhal S, Shrager JB, Rosenthal DI, LiVolsi VA, Kaiser LR (2003) Comparison of stages I-II thymoma treated by complete resection with or without adjuvant radiation. Ann Thorac Surg 76: 1635-1641.

4. Berman AT, Litzky L, Livolsi V, Singhal S, Kucharczuk JC, et al. (2011) Adjuvant radiotherapy for completely resected stage 2 thymoma. Cancer 117: 3502-3508.

5. Patel S, Macdonald OK, Nagda S, Bittner N, Suntharalingam M (2012) Evaluation of the role of radiation therapy in the management of malignant thymoma. Int J Radiat Oncol Biol Phys 82: 1797-1801.

6. Curran WJ Jr, Kornstein MJ, Brooks JJ, Turrisi AT 3rd (1988) Invasive thymoma: the role of mediastinal irradiation following complete or incomplete surgical resection. J Clin Oncol 6: 1722-1727.

7. Gomez D, Komaki R (2010) Technical advances of radiation therapy for thymic malignancies. J Thorac Oncol 5: S336-343. 